\section{Vacinação contra influenza em idosos por área de residência: prevalência e fatores associados}

\section{Influenza vaccination among the elderly according to place of residence: prevalence and associated factors}

Priscila Maria S. Bergamo Francisco'

Maria Rita Donalisio'

Marilisa Berti de Azevedo Barros ${ }^{1}$

Chester Luis Galvão Cesar ${ }^{2}$

Luana Carandina ${ }^{3}$

Moisés Goldbaum ${ }^{4}$

'Depto de Medicina Preventiva e Social, Faculdade de Ciências Médicas UNICAMP

${ }^{2}$ Depto de Epidemiologia, Faculdade de Saúde Pública - USP

${ }^{3}$ Depto de Medicina em Saúde Pública, Faculdade de Medicina de Botucatu UNESP

${ }^{4}$ Depto de Medicina Preventiva, Faculdade de Medicina - USP

O estudo foi financiado pela Fundação de Amparo a Pesquisa do Estado de São Paulo (FAPESP Processo n. 14099-7), Secretaria Estadual de Saúde de São Paulo e auxílio produtividade pelo Conselho Nacional de Desenvolvimento Científico e Tecnológico (CNPq). Francisco PMSB foi bolsista pela Coordenação de Aperfeiçoamento de Pessoal Nível Superior (CAPES).

Correspondência: Maria Rita Donalisio. DMPS/FCM/UNICAMP Caixa Postal 6111 - Campinas SP Cep: 13.083-970. Email: donalisi@fcm.unicamp.br

\section{Resumo}

A vacinação contra influenza é a principal forma de prevenir e reduzir a morbidade e mortalidade associadas à doença entre os idosos e grupos de risco. O objetivo deste estudo é determinar fatores demográficos, socioeconômicos, comportamentais e de saúde associados à vacinação, entre idosos residentes em diferentes áreas do Estado de São Paulo, no período de 2001 a 2002. Trata-se de um delineamento transversal de base populacional que considerou os idosos residentes em duas áreas do Estado: uma composta pelo município de Campinas e distrito do Butantã, na cidade de São Paulo, e outra pelos municípios de Taboão da Serra, Embu e Itapecerica da Serra (região metropolitana do município de São Paulo). A amostra foi composta por 849 e 641 indivíduos com 60 anos ou mais, residentes em tais localidades, respectivamente. $\mathrm{Na}$ análise bruta foram utilizadas razões de prevalência e intervalos de confiança de $95 \%$ e a análise multivariada foi realizada pela regressão de Poisson. A prevalência de vacinação auto-referida foi de $66,9 \%$ entre os residentes em Campinas e no distrito do Butantã e 67,6\% naqueles das demais localidades. Após análise ajustada, para os idosos de Campinas e Butantã, apenas menor escolaridade (RP = 1,25; IC 95\%: 1,02-1,54) esteve associada à vacinação. Já na área composta pelos municípios menos populosos, idade mais avançada ( $\mathrm{RP}=1,15$; IC 95\%: 1,02-1,31), hipertensão arterial (RP = 1,21; IC 95\%: 1,02$1,45)$, diabetes ( $\mathrm{RP}=1,16$; IC 95\%: 1,01-1,33) e doença crônica de pulmão (RP = 1,30; IC 95\%: 1,03-1,64) referidas, estiveram também associadas. Apesar de a prevalência de vacinação contra influenza entre os idosos das diversas localidades ser praticamente a mesma, pôde-se observar diferenças no perfil do idoso quanto à referência desse procedimento preventivo.

Palavras-chave: Vacinação contra influenza. Prevalência. Estudos tranversais. Idoso. 


\section{Abstract}

Influenza vaccination is the main means of preventing and reducing morbidity and mortality associated with this disease among the elderly population. The aim of the present study is to determine demographic, socioeconomic, behavioral, and health-related factors associated with vaccination among the elderly living in different areas of the State of Sao Paulo, in the 2001-2002 period. It is a cross-sectional population-based study including elderly subjects living in two areas of the State: one comprises the municipality of Campinas and the Butantã district in the City of São Paulo, and another comprises the municipalities of Taboão da Serra, Embu, and Itapecerica da Serra (metropolitan area of the City of Sao Paulo). The samples included 849 and 641 subjects, aged 60 years or older living in the two areas, respectively. Crude analysis was carried out using prevalence ratios and $95 \%$ confidence intervals. Multiple analysis was performed by Poisson regression. The prevalence of self-reported vaccination was $66.9 \%$ among residents of Campinas and in the Butantã district, and $67.6 \%$ among residents of the remaining cities. After adjusted analysis, among the subjects living in Campinas and in the Butantã district, only lesser schooling ( $\mathrm{PR}=1.25$; 95\%CI: 1.02-1.54) was associated with vaccination. In the group comprising the less densely populated municipalities, older age $(\mathrm{PR}=$ 1.15; 95\%CI: 1.02-1.31), and reported hypertension ( $\mathrm{PR}=1.21$; 95\%CI: 1.02-1.45), diabetes ( $\mathrm{PR}=1.16$; 95\%CI: 1.01-1.33), and chronic pulmonary disease $(\mathrm{PR}=1.30$; $95 \%$ CI: 1.03-1.64) were also associated with the outcome. Despite the prevalence of influenza vaccination among the elderly populations in the different areas being practically identical, we found differences in the profile of elderly individuals with respect to reported vaccination.

Keywords: Influenza vaccination. Prevalence. Cross-sectional studies. Elderly.

\section{Introdução}

Todos os anos, a influenza e suas complicações são responsáveis por excesso de hospitalizações, mortes e gastos com serviços de saúde em todo o mundo. A maioria dos quadros graves desencadeados pela doença ocorre entre idosos e em pessoas portadoras de doenças crônicas ${ }^{1-4}$. Vários estudos têm apontado a vacinação contra influenza como um meio efetivo de prevenção e redução da morbidade e mortalidade associadas à doença entre os idosos e grupos de risco, ou seja, os benefícios decorrentes desse procedimento preventivo estão amplamente estabelecidos ${ }^{1-5}$.

Embora haja recomendação para vacinação anual contra influenza nesses gru$\operatorname{pos}^{4,6}$, a adesão a essa prática varia entre os idosos residentes em diferentes localidades. Na Itália, em 1995, as taxas de vacinação foram de $26 \%, 32 \%$ e $48,6 \%$ entre os idosos residentes em Milão, Molise e Nápoles, respectivamente ${ }^{7}$. Na Alemanha, o percentual de imunização foi de $37 \%$ e $55 \%$ entre pessoas de 60 anos e mais das antigas Alemanha Ocidental e Oriental, durante a estação de $1999-2000^{8}$. Na Inglaterra, $65 \%$ e $68 \%$ dos idosos de 65 anos e mais foram vacinados durante as estações de 2000/2001 e 2001/2002; no entanto, as coberturas variaram entre as regiões do país ${ }^{9}$. Na Espanha, Puig-Barbera et al. (1999) ${ }^{10}$ encontraram níveis que oscilaram entre $60 \%$ a $84 \%$. Nos Estados Unidos, em 2002, $66,4 \%$ dos idosos com 65 anos e mais referiram a vacinação nos últimos 12 meses e os percentuais variaram de $32,2 \%$ nas Ilhas Virgens a 76,6\% em Minesota ${ }^{11}$. No Brasil, em 1999, 87,3\% dos idosos de 65 anos e mais foram vacinados contra influenza. No período de 2000 a 2003 ampliou-se a oferta da vacina para os maiores de 60 anos; no entanto, houve uma redução dos níveis de cobertura. No Estado de São Paulo, o percentual de vacinados no período foi de $65 \%{ }^{12}$. Em 2004, o país retomou os níveis preconizados pela Organização Mundial da Saúde (OMS) e vacinou $85 \%$ da população idosa (cerca de 13 milhões de pesso- 
as) e 95\% dos municípios brasileiros alcançaram a meta $(70 \%)^{6}$.

Apesar da disponibilização da vacina pelo Ministério da Saúde em âmbito nacional, mesmo no Estado de São Paulo, onde as coberturas vacinais estiveram acima da meta estabelecida em 2004, ainda existem municípios com coberturas insatisfatórias, revelando que os fatores individuais determinantes da adesão à vacinação podem diferir segundo grupos específicos e locais de residência. O conhecimento dos fatores associados à vacinação é importante na medida em que pode embasar ações tanto para o esclarecimento dos benefícios da vacina quanto para o aumento da cobertura em subgrupos da população idosa e em localidades específicas.

O objetivo deste estudo é determinar fatores demográficos, socioeconômicos, comportamentais e de saúde associados à vacinação, entre idosos residentes em diferentes áreas do Estado de São Paulo no período de 2001 a 2002.

\section{Métodos}

Neste trabalho foram utilizados dados do estudo multicêntrico - "Inquérito de saúde de base populacional em municípios de estado de São Paulo” (ISA-SP), realizado pelas universidades públicas paulistas (USP, UNESP e UNICAMP) com a parceria da Secretaria Estadual da Saúde de São Paulo em municípios do Estado, no período de 2001 a $2002^{13}$. A pesquisa teve como base uma amostra probabilística da população: estratificada, por conglomerados e obtida em dois estágios de seleção. No primeiro estágio foram amostrados setores censitários; no segundo, foi selecionada uma amostra sistemática simples de domicílios de cada setor. Estimou-se um tamanho mínimo de amostra de 192 pessoas para cada domínio de idade e sexo, tendo como base a estimativa de prevalência de 50\% com nível de confiança de $95 \%$, erro máximo de 0,10 e efeito do delineamento (deff) igual a 2. Considerandose os possíveis efeitos da não resposta ao redor de $20 \%$, o tamanho da amostra foi corrigido para 250 indivíduos em cada domínio.

Todos os participantes do ISA-SP com 60 anos ou mais residentes nos municípios de Taboão da Serra, Embu, Itapecerica da Serra, São Paulo (Butantã) e Campinas, com respostas válidas para vacinação contra influenza $(n=1.443)$ foram selecionados para o presente trabalho, que subdividiu a população idosa em duas áreas de residência: área 1, composta por Campinas e distrito do Butantã ( $\mathrm{n}=824)$; e área 2, pelos demais municípios $(n=619)$. A alocação dos municípios nas áreas foi baseada no tamanho da população residente e nas características sociodemográficas dos mesmos.

A variável dependente deste estudo é a vacinação contra influenza auto-referida nos 12 meses que antecederam a pesquisa (sim ou não). As variáveis independentes analisadas foram características demográficas e socioeconômicas, comportamentais, indicadores da condição de saúde e uso de medicamentos. As características demográficas e socioeconômicas consideradas foram: sexo, idade, situação conjugal, escolaridade e renda per capita. As comportamentais incluíram ingestão de bebida alcoólica, tabagismo e índice de massa corporal $(\mathrm{IMC}=\mathrm{Kg} / \mathrm{m} 2)$, calculado com dados de peso e altura referidos. Entre os indicadores da condição de saúde foram considerados a percepção atual da própria saúde; internação hospitalar nos últimos 12 meses; número de doenças crônicas; relato de doenças específicas, a saber: hipertensão, diabetes, artrite/reumatismo/artrose, doença renal crônica, doença do coração e doença crônica de pulmão (asma/bronquite/enfisema); e uso de medicamentos.

As análises dos dados foram conduzidas utilizando-se o programa Stata 7.0, cujos procedimentos para análise de inquéritos populacionais incorporam as ponderações necessárias, produzindo melhor estimativa do erro padrão, o qual é influenciado pela conglomeração das unidades e estratificação derivadas do delineamento amostral complexo ${ }^{14}$. A análise bivariada foi rea- 
lizada utilizando-se como estimadores as razões de prevalência e intervalos de confiança de $95 \%$. A análise multivariada foi feita por meio de regressão de Poisson, utilizando-se o "backward selection" disponível para análise múltipla em inquéritos no programa Stata, que incluiu todas as variáveis que apresentaram o valor de $\mathrm{p}<0,20$ na análise bivariada. Como as variáveis escolaridade e renda per capita estiveram altamente associadas, apenas a escolaridade foi incluída no modelo múltiplo ${ }^{15}$. Também a variável "uso de medicamentos" não foi considerada na análise multivariada, dada a sua forte associação com a presença de doença crônica.

O projeto deste estudo foi aprovado pelo Comitê de Ética em pesquisa da Faculdade de Ciências Médicas da UNICAMP sob o parecer no $369 / 2000$.

\section{Resultados}

Comparando as regiões é possível observar perfis diferenciados entre elas com relação a algumas características demográficas e socioeconômicas. A Tabela 1 apresenta as características da amostra de idosos estudada, segundo área de residência. A área 1, composta por Campinas e distrito do Butantã, apresentou estrutura etária mais idosa. No que se refere à escolaridade, houve uma diferença marcante, já que a porcentagem de idosos com nove ou mais anos de estudo foi cerca de quatro vezes menor entre os residentes na área 2, composta pelos municípios menos populosos. Entre os que nunca freqüentaram escola, 11,6\% e 23,6\% pertenciam às áreas 1 e 2, respectivamente (dados não apresentados). A distribuição da renda familiar per capita também diferiu entre as áreas, já que cerca de 55,2 \% (IC 50,3-60,1) dos idosos residentes na área 2 apresentaram renda inferior ou igual a um salário mínimo e apenas 10,5\% (IC 8,2 - 12,7) mostraram melhor condição econômica (renda superior a 3 salários), sendo essa proporção cerca de três vezes maior entre os residentes na área 1.
A proporção de não fumantes e dos que referiram boa percepção da saúde foi maior entre os idosos residentes nos municípios de Campinas e no distrito do Butantã, em São Paulo, porém estes também apresentaram maior ingestão regular de álcool e uso de medicamentos. Quanto às demais características, as proporções foram semelhantes em ambas as áreas. A prevalência de vacinação contra influenza auto-referida no período estudado foi de $66,9 \%$ (IC 64,5 - 69,3) e $67,6 \%$ (IC 65,7 - 69,5) entre os residentes nas áreas 1 e 2, respectivamente (Tabela 1).

As prevalências de vacinação segundo as variáveis demográficas, socioeconômicas, comportamentais e de saúde selecionadas nesse estudo estão apresentadas na Tabela 2. A probabilidade de vacinação aumentou com a idade em ambas as áreas. No município de Campinas e distrito do Butantã também foi possível observar, na análise bivariada, aumento significativo da prevalência nos de menor escolaridade, naqueles com renda inferior ou igual a um salário mínimo e entre os que referiram uso de medicamento nos três dias anteriores à pesquisa. Na área 2, composta por municípios menores, verificaram-se prevalências significativamente mais elevadas nos que referiram uso de medicamentos e nos portadores de hipertensão arterial, diabetes e doença crônica de pulmão.

Após análise ajustada pode-se observar diferenças nos preditores de vacinação, segundo local de residência. Entre os idosos residentes no município de Campinas e distrito de Butantã, apenas menor escolaridade ( $\mathrm{RP}=1,25$; $\mathrm{IC}=1,02$ - 1,54) esteve associada à referência de vacinação. Já nos municípios de Taboão da Serra, Embu e Itapecerica da Serra, foi encontrada associação independente para idade $(\mathrm{RP}=1,15$; $\mathrm{IC}=1,02$ - 1,31), hipertensão arterial (RP = 1,21 ; IC = 1,02-1,45), diabetes ( $\mathrm{RP}=1,16$; IC $=1,01-1,33)$ e doença crônica de pulmão $(\mathrm{RP}=1,30 ; \mathrm{IC}=1,03-1,64)$ referidas (Tabela 3$)$. 
Tabela 1 - Descrição da amostra de idosos segundo características demográficas, socioeconômicas, comportamentais e de saúde por área de residência. ISA-SP, 2001-2002. Table 1 - Description of the sample according to demographic, socioeconomic, behavioral, and health-related characteristics according to area of residence. ISA-SP, 2001-2002.

\begin{tabular}{|c|c|c|c|c|}
\hline \multirow[t]{2}{*}{ Variáveis e Categorias } & \multicolumn{2}{|r|}{ Área 1} & \multicolumn{2}{|c|}{ Área 2} \\
\hline & $\mathrm{N}$ & $(\%)$ & $\mathrm{N}$ & $(\%)$ \\
\hline \multicolumn{5}{|l|}{ Sexo } \\
\hline Masculino & 408 & $42,6(39,2-46,0)$ & 282 & $43,0(39,7-46,3)$ \\
\hline Feminino & 416 & $57,4(53,2-61,6)$ & 337 & $57,0(53,4-60,6)$ \\
\hline \multicolumn{5}{|l|}{ Faixa etária } \\
\hline 60 a 69 & 459 & $54,2(49,7-58,8)$ & 376 & $65,5(60,6-70,4)$ \\
\hline 70 e mais & 365 & $45,8(41,6-49,9)$ & 243 & $34,5(30,1-38,9)$ \\
\hline \multicolumn{5}{|l|}{ Situação conjugal } \\
\hline Com cônjuge & 505 & $58,6(54,4-62,8)$ & 331 & $55,4(51,1-59,8)$ \\
\hline Sem cônjuge & 317 & $41,4(37,8-45,0)$ & 286 & $44,6(39,9-49,3)$ \\
\hline \multicolumn{5}{|l|}{ Escolaridade } \\
\hline Menos de 9 anos & 654 & $78,5(74,0-83,1)$ & 539 & $94,9(90,8-99,1)$ \\
\hline 9 anos ou mais & 164 & $21,5(18,2-24,8)$ & 79 & $5,1(3,5-6,6)$ \\
\hline \multicolumn{5}{|l|}{ Renda per capita (SM) } \\
\hline$<=1$ salário mínimo & 228 & $23,7(21,1-26,3)$ & 245 & $55,2(50,3-60,1)$ \\
\hline >1 a 3 salários mínimos & 324 & $41,0(37,7-44,3)$ & 232 & $34,3(29,8-38,8)$ \\
\hline > 3 salários mínimos & 272 & $35,3(31,5-39,1)$ & 142 & $10,5(8,2-12,7)$ \\
\hline \multicolumn{5}{|l|}{ Ingestão de álcool } \\
\hline Ocasional/não ingere & 600 & $73,0(68,9-77,2)$ & 501 & $84,0(79,4-88,7)$ \\
\hline Regular & 224 & $27,0(23,9-30,1)$ & 118 & $16,0(13,0-18,9)$ \\
\hline \multicolumn{5}{|l|}{ Tabagismo } \\
\hline Não fumante & 720 & $89,4(84,7-94,0)$ & 512 & $81,0(76,4-85,6)$ \\
\hline Ex-fumante e fumante & 101 & $10,6(8,8-12,4)$ & 106 & $19,0(15,6-22,4)$ \\
\hline \multicolumn{5}{|l|}{ Percepção da própria saúde } \\
\hline Excelente/muito boa/boa & 686 & $88,3(83,6-92,9)$ & 499 & $79,5(74,9-84,1)$ \\
\hline Ruim/muito ruim & 103 & $11,7(9,8-13,7)$ & 111 & $20,5(17,0-24,1)$ \\
\hline \multicolumn{5}{|l|}{ Uso de medicamento } \\
\hline Não & 208 & $21,5(18,9-24,1)$ & 188 & $32,5(29,0-36,1)$ \\
\hline Sim & 614 & $78,5(74,3-82,7)$ & 431 & $67,5(62,7-72,2)$ \\
\hline \multicolumn{5}{|l|}{ Internação hospitalar } \\
\hline Nenhuma & 730 & $89,1(84,4-93,8)$ & 546 & $88,0(83,4-92,5)$ \\
\hline Uma ou mais & 93 & $10,9(9,4-12,4)$ & 73 & $12,0(9,2-14,9)$ \\
\hline \multicolumn{5}{|l|}{ Número de doenças crônicas } \\
\hline Nenhuma & 101 & $12,1(10,2-14,0)$ & 69 & $10,7(8,0-13,4)$ \\
\hline Uma ou mais & 683 & $87,9(83,2-92,6)$ & 513 & $89,3(84,2-94,4)$ \\
\hline \multicolumn{5}{|c|}{ Relato das seguintes doenças/condições crônicas ${ }^{1}$} \\
\hline Hipertensão & 409 & $51,1(47,3-54,8)$ & 303 & $52,7(48,0-57,4)$ \\
\hline Diabetes & 131 & $15,5(13,4-17,6)$ & 95 & $15,5(12,5-18,4)$ \\
\hline Artrite/reumatismo/artrose & 215 & $27,6(24,9-30,3)$ & 155 & $23,9(20,3-27,6)$ \\
\hline Doença renal crônica & 52 & $4,3(3,2-5,4)$ & 31 & $4,3(2,7-5,9)$ \\
\hline Doença do coração & 126 & $16,7(14,6-18,7)$ & 80 & $11,8(8,9-14,8)$ \\
\hline $\begin{array}{l}\text { Doença crônica de pulmão (asma/ } \\
\text { bronquite/enfisema) }\end{array}$ & 57 & $6,8(5,4-8,2)$ & 43 & $6,9(5,2-8,6)$ \\
\hline Vacinação contra influenza & 824 & $66,9(64,5-69,3)$ & 619 & $67,6(65,7-69,5)$ \\
\hline
\end{tabular}

Área 1: Município de Campinas e distrito do Butantã (município de São Paulo)/Area 1: Muinicipality of Campinas and Butantã district (municipality of São Paulo);

Área 2: Municípios de Taboão da Serra, Embu e Itapecerica da Serra/Area 2: Muinicipalties of Taboão da Serra, Embu and Itapecerica da Serra.

a porcentagem de idosos vacinados em cada categoria/N: number of individuals in the non-weighted sample.

$\mathrm{SM}=$ Salário mínimo vigente à época da pesquisa: março a abril/2001 = R\$ 151,00; maio/2001 a março/2002 = R\$ 180,00 e abril a setembro/2002 = R\$ 200,00/MW=Minimum wage at the time of study:March toApril/2001 = R\$ 151.00; May/2001 to March/2002 $=R \$ 180.00$ and April to September $/ 2002=R \$ 20000$.

${ }^{1}$ Percentual dos que disseram sim (classe de referência: resposta negativa para doença/condição crônica)/'Percentage of subjects who answered 'yes' (reference class: negative answer for chronic diseases/conditions) 
Tabela 2 - Razão de prevalência bruta para vacinação contra influenza auto-referida em idosos, segundo área de residência. ISA-SP, 2001-2002.

Table 2 - Crude prevalence ratio for self-reported influenza vaccination among elderly subjects according to area of residence. ISA-SP, 2001-2002.

\begin{tabular}{|c|c|c|c|c|c|c|}
\hline \multirow[t]{2}{*}{ Variáveis e Categorias } & \multicolumn{3}{|c|}{ Área 1} & \multicolumn{3}{|c|}{ Área 2} \\
\hline & $(\%)^{a}$ & $\mathrm{RP}_{(\mathrm{IC} \text { 95\%) }}$ & $p$ valor & $(\%)^{a}$ & $\mathrm{RP}_{\text {(IC 95\%) }}$ & $\mathrm{p}$ valor \\
\hline \multicolumn{7}{|l|}{ Sexo } \\
\hline Masculino & 68,2 & 1 & & 66,2 & 1 & \\
\hline Feminino & 65,8 & $0,96(0,87-1,06)$ & $p=0,47$ & 68,6 & $1,04(0,91-1,18)$ & $p=0,58$ \\
\hline \multicolumn{7}{|l|}{ Faixa etária } \\
\hline 60 a 69 & 63,0 & 1 & & 64,1 & 1 & \\
\hline 70 e mais & 71,4 & $1,13(1,01-1,28)$ & $p=0,05$ & 74,1 & $1,15(1,02-1,30)$ & $p=0,02$ \\
\hline \multicolumn{7}{|l|}{ Situação conjugal } \\
\hline Com cônjuge & 66,9 & 1 & & 66,7 & 1 & \\
\hline Sem cônjuge & 68,0 & $1,03(0,93-1,14)$ & $p=0,55$ & 68,7 & $1,03(0,88-1,21)$ & $p=0,71$ \\
\hline \multicolumn{7}{|l|}{ Escolaridade } \\
\hline Menos de 9 anos & 70,1 & $1,25(1,02-1,54)$ & $p=0,03$ & 67,7 & $1,00(0,74-1,33)$ & $p=0,97$ \\
\hline 9 anos ou mais & 55,8 & 1 & & 67,4 & 1 & \\
\hline \multicolumn{7}{|l|}{ Renda per capita (SM) } \\
\hline <=1 salário mínimo & 75,8 & 1 & & 63,8 & 1 & \\
\hline >1 a 3 salários mínimos & 66,5 & $0,88(0,78-0,98)$ & $p=0,02$ & 75,0 & $1,18(0,98-1,41)$ & $p=0,07$ \\
\hline > 3 salários mínimos & 61,3 & $0,81(0,68-0,97)$ & $p=0,02$ & 63,2 & $0,99(0,78-1,26)$ & $p=0,94$ \\
\hline \multicolumn{7}{|l|}{ Ingestão de álcool } \\
\hline Ocasional/não ingere & 68,7 & 1 & & 68,8 & 1 & \\
\hline Regular & 61,9 & $0,90(0,79-1,03)$ & $p=0,11$ & 61,1 & $0,88(0,69-1,14)$ & $p=0,35$ \\
\hline \multicolumn{7}{|l|}{ Tabagismo } \\
\hline Não fumante & 67,3 & 1 & & 68,7 & 1 & \\
\hline Ex-fumante e fumante & 63,5 & $0,94(0,78-1,13)$ & $p=0,53$ & 63,3 & $0,92(0,75-1,13)$ & $p=0,42$ \\
\hline \multicolumn{7}{|l|}{ Percepção da própria saúde } \\
\hline Excelente/muito boa/boa & 67,6 & 1 & & 67,1 & 1 & \\
\hline Ruim/muito ruim & 63,8 & $0,94(0,80-1,12)$ & $p=0,50$ & 68,9 & $1,03(0,83-1,26)$ & $p=0,80$ \\
\hline \multicolumn{7}{|l|}{ Uso de medicamento } \\
\hline Não & 58,8 & 1 & & 59,7 & 1 & \\
\hline Sim & 69,1 & $1,17(1,01-1,36)$ & $p=0,03$ & 71,3 & $1,19(1,02-1,40)$ & $p=0,03$ \\
\hline \multicolumn{7}{|l|}{ Internação hospitalar } \\
\hline Nenhuma & 66,8 & 1 & & 67,1 & 1 & \\
\hline Uma ou mais & 67,2 & $1,01(0,87-1,16)$ & $p=0,93$ & 70,6 & $1,05(0,76-1,46)$ & $p=0,75$ \\
\hline \multicolumn{7}{|l|}{ Número de doenças crônicas } \\
\hline Nenhuma & 70,8 & 1 & & 56,6 & 1 & \\
\hline Uma ou mais & 66,7 & $0,94(0,80-1,11)$ & $p=0,47$ & 68,9 & $1,22(0,84-1,77)$ & $p=0,29$ \\
\hline \multicolumn{7}{|c|}{ Relato das seguintes doenças ${ }^{1}$} \\
\hline Hipertensão & 70,9 & $1,13(0,99-1,28)$ & $p=0,06$ & 74,8 & $1,24(1,04-1,49)$ & $p=0,02$ \\
\hline Diabetes & 74,2 & $1,13(0,98-1,31)$ & $p=0,10$ & 78,5 & $1,20(1,02-1,41)$ & $p=0,03$ \\
\hline Artrite/reumatismo/artrose & 71,5 & $1,10(0,97-1,26)$ & $p=0,14$ & 73,5 & $1,12(0,99-1,28)$ & $p=0,07$ \\
\hline Doença renal crônica & 65,3 & $0,98(0,75-1,27)$ & $p=0,85$ & 79,4 & $1,18(0,91-1,54)$ & $p=0,20$ \\
\hline Doença do coração & 67,9 & $1,02(0,89-1,17)$ & $p=0,77$ & 69,3 & $1,02(0,84-1,24)$ & $p=0,80$ \\
\hline Doença crônica de pulmão & 66,3 & $0,99(0,82-1,20)$ & $p=0,93$ & 83,2 & $1,25(1,04-1,52)$ & $p=0,02$ \\
\hline
\end{tabular}

Area 1: Municipality of Campinas and Butantã District (municipality of Sao Paulo)/ Área 1: Município de Campinas e distrito do Butantã (município de São Paulo); Area 2: Municipalities of Taboão da Serra, Embu, and Itapecerica da Serra/Área 2: Municípios de Taboão da Serra, Embu e Itapecerica da Serra.

* percentage of elderly subjects vaccinated in each category/N: número de indivíduos na amostra não ponderada.

$\mathrm{MW}=$ minimum wage at the time of the survey: March - April/2001 = R\$ 151.00; May/2001 - March /2002 = R\$ 180.00, and April - September/2002 = R\$ 200.00/SM=Salário mínimo vigente à época da pesquisa: março a abril/2001 $=R \$ 151,00 ;$ maio $/ 2001$ a março/2002 $=R \$ 180,00$ e abril a setembro/2002 = R\$ 200,00.

'Percentage of subjects who answered 'yes' (reference class: negative answer for chronic diseases/conditions)/ ${ }^{1}$ Percentual dos que disseram sim (classe de referência: resposta negativa para doença/condição crônica) 
Tabela 3 - Modelo de regressão multivariada de Poisson para vacinação contra influenza auto-referida, por área de residência. ISA-SP, 2001-2002.

Table 3 - Poisson multivariate regression model for self-reported influenza vaccination, according to area of residence. ISA-SP, 2001-2002.

\begin{tabular}{|c|c|c|c|c|c|}
\hline Variáveis e categorias & $\mathrm{RP}^{1}$ & Erro-padrão & IC $95 \%{ }^{2}$ & Valor de $\mathrm{p}$ & Deff $^{3}$ \\
\hline \multicolumn{6}{|c|}{ Área 1: Campinas e distrito do Butantã } \\
\hline \multicolumn{6}{|c|}{ Escolaridade } \\
\hline Nove anos ou mais & 1 & & & & \\
\hline Menos de 9 anos & 1,25 & 0,13 & $(1,02-1,54)$ & 0,03 & 2,07 \\
\hline \multicolumn{6}{|c|}{ Área 2: Taboão da Serra, Embu, Itapecerica da Serra } \\
\hline \multicolumn{6}{|c|}{ Faixa etária } \\
\hline 60 a 69 anos & 1 & & & & \\
\hline 70 anos ou mais & 1,15 & 0,07 & $(1,02-1,31)$ & 0,03 & 1,25 \\
\hline \multicolumn{6}{|l|}{ Hipertensão arterial } \\
\hline Não & 1 & & & & \\
\hline Sim & 1,21 & 0,11 & $(1,02-1,45)$ & 0,03 & 2,30 \\
\hline \multicolumn{6}{|l|}{ Diabetes } \\
\hline Não & 1 & & & & \\
\hline Sim & 1,16 & 0,08 & $(1,01-1,33)$ & 0,04 & 1,17 \\
\hline \multicolumn{6}{|c|}{ Doença crônica de pulmão } \\
\hline Não & 1 & & & & \\
\hline Sim & 1,30 & 0,15 & $(1,03-1,64)$ & 0,03 & 1,83 \\
\hline
\end{tabular}

'Prevalence ratio/'Razão de prevalência

${ }^{2} 95 \%$ confidence interval/2Intervalo de confiança em nível de 95\%

${ }^{3}$ Design effect $/{ }^{3}$ Efeito do desenho

\section{Discussão}

As coberturas vacinais contra influenza foram semelhantes entre os idosos residentes nas diferentes regiões analisadas (66,9\% e 67,6\%, respectivamente) e estiveram abaixo da meta estabelecida pelo $\mathrm{Mi}$ nistério da Saúde no período estudado, assim como na maioria dos municípios do Estado de São Paulo ${ }^{16}$.

Dentre as limitações do estudo deve-se considerar que os dados foram auto-referidos, não havendo confirmação objetiva por meio de registro em carteira de vacinação. No entanto, alguns autores têm destacado a validade e acurácia do recordatório do idoso quanto à informação auto-referida de vacinação contra influenza ${ }^{17,18}$.

Os dados avaliados nesse estudo não foram obtidos de um inquérito específico para avaliar comportamentos preventivos; no entanto, referem-se a inquérito amplo que abordou condições e estilo de vida, percepção e qualidade de saúde, morbi- dade referida, uso de serviços de saúde e consumo de medicamentos, incluindo domínios segundo faixas etárias e abrangendo a população de 60 anos e mais ${ }^{13}$.

Estimativas obtidas a partir de amostragem por conglomerados com qualquer grau de complexidade são menos precisas, já que costumam reduzir a heterogeneidade da amostra em relação às variáveis de interesse, tornando necessária a utilização de rotinas especiais de estimação e de testes de hipóteses que considerem o delineamento amostral ${ }^{14}$.

Em estudos transversais com desfechos binários, a regressão logística é amplamente utilizada; entretanto, a medida de efeito neste modelo é a razão de chances e não a razão de prevalências, medida natural desse delineamento epidemiológico. Em situações em que a prevalência é alta, a razão de chances superestima a razão de prevalências, podendo distorcer a interpretação dos resultados ${ }^{19}$. Isso deve ser considerado, na medida em que a identificação dos fato- 
res associados à vacinação contra influenza pode subsidiar ações de saúde pública para o esclarecimento dos benefícios da vacina e aumento das taxas de cobertura em subgrupos e localidades específicas. Desta forma, optou-se pela utilização da regressão de Poisson que estima diretamente as razões de prevalência ${ }^{20}$.

Os resultados indicam que a adesão à vacinação foi maior entre os idosos de 70 anos ou mais em ambas as áreas, fato também observado por outros autores ${ }^{7,16,21}$. No entanto, neste estudo a idade somente pôde ser considerada como fator independente associado à vacinação entre os idosos residentes nos municípios menos populosos. Não houve associação estatística significativa entre as variáveis comportamentais selecionadas nesse estudo e a vacinação, assim como foi verificado entre idosos ingleses maiores de 65 anos $^{22}$.

Os idosos que referiram uso de medicamentos nos três dias anteriores à pesquisa apresentaram maior probabilidade de vacinação na análise bruta, em ambas as áreas. Essa variável pode ser indicadora de doença crônica não referida, mas também pode estar relacionada a quadros agudos e automedicação. Considerando a associação entre o uso de medicamentos e presença de doença crônica encontrada nesse estudo ( $p<0,001)$, essa variável não foi incluída no modelo múltiplo.

A proporção de idosos com nove ou mais anos de estudo em Campinas e no Butantã foi cerca de quatro vezes maior quando comparada às demais localidades e, quanto maior a escolaridade do idoso, menor a adesão à vacinação. Devido à forte associação encontrada entre escolaridade e renda per capita (dados não apresentados), é possível supor que os idosos com maior escolaridade também tenham acesso diferenciado aos serviços de saúde, medicamentos, planos e convênios, buscando soluções para seus problemas de saúde em serviços privados ou conveniados. Porém estes serviços indicam pouco a vacinação, como apontam alguns autores ${ }^{16,25}$. A divulgação dos benefícios da vacinação pode não abranger com intensidade esse grupo de idosos, com melhor condição econômica e de escolaridade em grandes cidades, supondo que a maior adesão destes indivíduos tem sido relacionada com a recomendação médica, como apontam vários estudos ${ }^{8,16,22,23,24}$.

Apenas nas localidades menos populosas, hipertensão arterial, diabetes e doença crônica de pulmão, auto-referidas, também estiveram independentemente associadas à vacinação. Tanto os idosos quanto demais pessoas com co-morbidades associadas estão incluídas nos grupos prioritários para vacinação ${ }^{4}$. No entanto, principalmente aqueles com melhores condições econômicas recebem cuidados de médicos de especialidades clínicas, que com menor freqüência recomendam a vacinação para seus pacientes, como apontam Nichol e Zimmermam (2001) em estudo sobre o conhecimento, atitudes e práticas de generalistas e especialistas nos Estados Unidos da América, com relação à indicação da vacinação em pacientes de alto risco $^{25}$.

Neste estudo verificou-se que nos municípios de Taboão da Serra, Embu e Itapecerica da Serra, a renda per capita dos idosos foi marcadamente inferior àquela observada entre os residentes em Campinas ou no distrito do Butantã. É possível que os idosos residentes em localidades menores sejam em sua maioria dependentes do sistema público de saúde, especialmente dos medicamentos distribuídos pelas unidades para suas enfermidades crônicas nos programas de hipertensão arterial, diabetes e outras doenças. Conseqüentemente, ocorre maior aproximação desse idoso aos serviços e, habituado a freqüentar a unidade básica de saúde, está mais atento às recomendações educacionais e ações preventivas, comparecendo com maior freqüência às campanhas vacinais.

Sabe-se que a grande maioria dos idosos apresenta pelo menos uma doença crônica, o que em geral os leva a redobrar a atenção com sua saúde. Deste modo, a recomendação da vacinação pelos profis- 
sionais de saúde é fundamental, independente de onde o idoso busque cuidados para seus problemas, para reduzir a morbidade e mortalidade associadas à infecção por influenza.

\section{Conclusões}

Apesar da prevalência de vacinação contra influenza entre os idosos das áreas estudadas ser praticamente a mesma, pôde-se observar diferenças no perfil do idoso quanto à referência desse procedimento preventivo.

Este estudo identificou subgrupos entre os idosos com maior probabilidade de não adesão à vacinação nas diversas áreas, que devem ser objeto de campanhas específicas para aumentar as coberturas vacinais. Dentre eles destacam-se os idosos com maior nível educacional e os portadores de doenças crônicas, particularmente em cidades maiores, bem como todos os indivíduos de 60 a 69 anos.

\section{Referências}

1. Hak E, Van Essen GA, Buskens E, Stalman W, Melker RA. Is immunising all patients with chronic lung disease in the community against influenza cost effective? Evidence from a general practice based clinical prospective cohort study in Utrecht, the Netherlands. J Epidemiol Community Health 1998; 52: 120-5.

2. Nichol KL, Wuorenma J, Von Sternberg T. Benefits of influenza vaccination for low-intermediate-, and highrisk senior citizens. Arch Intern Med 1998; 158: 1769-76.

3. Nichol KL, Nordin J, Mullooly J, Lask R, Filbrandt K, Iwane $\mathrm{M}$. Influenza vaccination and reduction in hospitalizations for cardiac disease and stroke among the elderly. NEngl J Med 2003; 348: 1322-32.

4. Harper SA, Fukuda K, Uyeki TM, Cox NJ, Bridges CB. Prevention and control of influenza: recommendations of the Advisory Committee on Immunization Practices (ACIP). MMWR Recomm Rep 2004; 53: 1-40.

5. Gross PA, Hermogenes AW, Sacks HS, Lau J, Levandowski RA. The efficacy of influenza vaccine in elderly persons. A meta-analysis and review of the literature. Ann Intern Med 1995; 123: 517-28.

6. Brasil. Ministério da Saúde. Secretaria de Vigilância em Saúde. Campanha Nacional de Vacinação do Idoso. Boletim Eletrônico Epidemiológico 2005.

7. Pregliasco F, Sodano L, Mensi C, Selvaggi MT, Adamo B, D'Argenio $P$ et al. Influenza vaccination among the elderly in Italy. Bull World Health Organ 1999; 77: 127-31.

8. Rehmet S, Ammon A, Pfaff G, Bocter N, Petersen LR. Cross-sectional study on influenza vaccination, Germany, 1999-2000. Emerging Infectious Diseases 2002; 8(12): $1442-7$.

9. Joseph C, Goddard N. Influenza vaccine uptake in the elderly: results from a rapid assessment of the effectiveness of new government policy in England for the winters 2000/2001 and 2001/2002. Vaccine 2003; 21: 1137-48.
10. Puig-Barbera J, Ors ZP, Vilchez PC, Lloria PF. Impacto de distintas estrategias en las tasas de vacunación antigripal en ancianos. Aten Primaria 1999; 23:339-45.

11. CDC. Public health and aging: influenza vaccination coverage among adults aged $>$ or $=50$ years and pneumococcal vaccination coverage among adults aged $>$ or $=65$ years - United States, 2002. MMWR Morb Mortal Wkly Rep 2003 Oct 17; 52(41): 987-92.

12. São Paulo. Secretaria de estado de Saúde de São Paulo Centro de Vigilância Epidemiológica "Prof. Alexandre Vranjac". Campanha Nacional de Vacinação contra Influenza 2005. São Paulo, SES, 2005 (Informe Técnico).

13. Cesar CLG; Carandina, L; Goldbaum, M; Barros MBA. Inquérito de saúde de base populacional em municípios do Estado de São Paulo (ISA-SP). Projeto submetido à FAPESP, 1998.

14. Pessoa DGC, Silva PLN, Duarte RPN. Análise estatística de dados de pesquisas por amostragem: problemas no uso de pacotes-padrão. Revista Brasileira de Estatística 1997; 58: 53-75.

15. Becket M. Converging health inequalities in later life - an artifact of mortality selection? J Health and Soc Behav 2000; 41: 106-19.

16. São Paulo. Secretaria do Estado de São Paulo, Centro de Referência em Saúde do Trabalhador de São Paulo. Pesquisas indicam pequena participação dos médicos no incentivo à vacinação contra influenza. Rev Saúde Pública 2004; 38(4): 607-8.

17. Mac Donald R; Baken L; Nelson A; Nichol KL. Validation of self-report of influenza and pneumococcal vaccination status in elderly outpatients. Am J Prev Med 1999; 16(3): 173-7.

18. Bedford D, Howell F. Influenza vaccination uptake in 1999 and older persons recall of vaccination. Ir Med J 2001; 94(9): 264-5. 
19. Hirakata VN. Alternativas de análise para um desfecho binário em estudos transversais e longitudinais

[dissertação de mestrado]. Pelotas: Universidade Federal de Pelotas; 1999.

20. Barros AJ, Hirakata VN. Alternatives for logistic regression in cross-sectional studies: an empirical comparison of models that directly estimate the prevalence ratio. $B M C$ Med Res Methodol 2003; 3(1): 21.

21. Sarriá A, Timoner J. Determinantes de la vacunatión de la gripe en personas mayores de 65 años. Rev Esp Salud Pública 2002; 76(1): 17-26.

22. Burns VE, Ring C, Carroll D. Factors influencing influenza vaccination uptake in an elderly, community-based sample. Vaccine 2005; 23(27): 3604-8.
23. Bovier PA, Chamot E, Gallacchi MB, Loutan L. Importance of patients' perceptions and general practitioners' recommendations in understanding missed opportunities for immunisations in Swiss adults. Vaccine 2001; 19: 4760-7.

24. Moura M, Silva LJ. Pesquisa de opinião sobre as campanhas de vacinação contra a influenza no estado de São Paulo. Boletim Epidemiológico Paulista 2004; 1: 8-10.

25. Nichol KL, Zimmerman R. Generalist and subspecialist physicians' knowledge, attitudes, and practices regarding influenza and pneumococcal vaccinations for elderly and other high-risk patients: a nationwide survey. Arch Intern Med 2001; 161(22): 2702-8.

Recebido em: 22/08/06 Versão reformulada reapresentada em: 15/03/06 Aprovado em: 16/04/06 